Provided for non-commercial research and educational use only. Not for reproduction or distribution or commercial use.

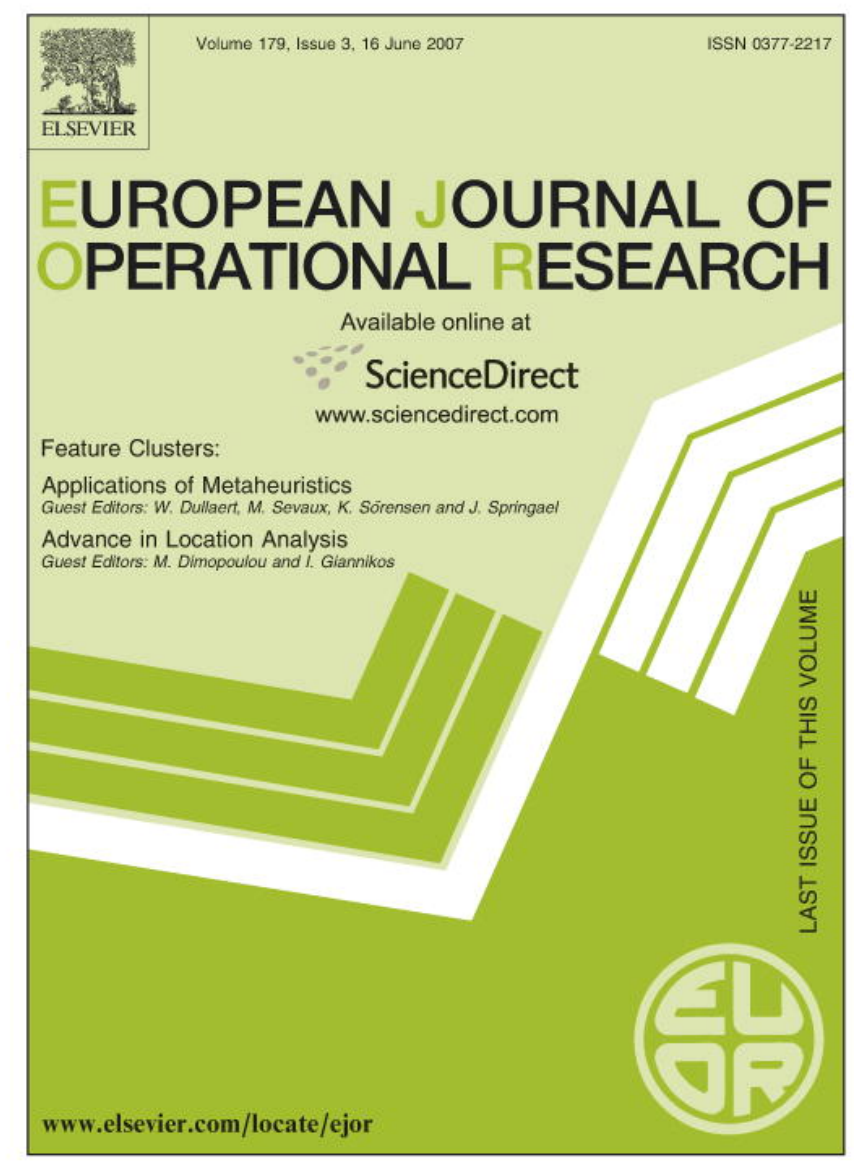

This article was originally published in a journal published by Elsevier, and the attached copy is provided by Elsevier for the author's benefit and for the benefit of the author's institution, for non-commercial research and educational use including without limitation use in instruction at your institution, sending it to specific colleagues that you know, and providing a copy to your institution's administrator.

All other uses, reproduction and distribution, including without limitation commercial reprints, selling or licensing copies or access,

or posting on open internet sites, your personal or institution's website or repository, are prohibited. For exceptions, permission may be sought for such use through Elsevier's permissions site at: 


\title{
Placing a finite size facility with a center objective on a rectangular plane with barriers
}

\author{
Avijit Sarkar, Rajan Batta, Rakesh Nagi \\ Department of Industrial Engineering, 420 Bell Hall, University at Buffalo (SUNY), Buffalo, NY 14260, USA
}

Received 15 December 2003; accepted 15 August 2005

Available online 4 April 2006

\begin{abstract}
This paper addresses the finite size 1-center placement problem on a rectangular plane in the presence of barriers. Barriers are regions in which both facility location and travel through are prohibited. The feasible region for facility placement is subdivided into cells along the lines of Larson and Sadiq [R.C. Larson, G. Sadiq, Facility locations with the Manhattan metric in the presence of barriers to travel, Operations Research 31 (4) (1983) 652-669]. To overcome complications induced by the center (minimax) objective, we analyze the resultant cells based on the cell corners. We study the problem when the facility orientation is known a priori. We obtain domination results when the facility is fully contained inside 1,2 and 3-cornered cells. For full containment in a 4-cornered cell, we formulate the problem as a linear program. However, when the facility intersects gridlines, analytical representation of the distance functions becomes challenging. We study the difficulties of this case and formulate our problem as a linear or nonlinear program, depending on whether the feasible region is convex or nonconvex. An analysis of the solution complexity is presented along with an illustrative numerical example.
\end{abstract}

(c) 2006 Elsevier B.V. All rights reserved.

Keywords: 1-Center placement; Finite size facility location; Barrier; Rectangular plane

\section{Introduction}

Location problems which impose restrictions on locating new facilities and/or travel through are typically referred to as constrained or restricted location problems. Such problems have the following two topographical properties: (1) the new facilities cannot be located within certain predescribed restricted areas in the plane; (2) it is not always necessary that

\footnotetext{
${ }^{*}$ Corresponding author. Fax: +17166453302.

E-mail address: nagi@buffalo.edu (R. Nagi).
}

any two points in the plane would be "simply communicating", i.e., the minimum travel distance between any two points in the plane may be made longer by the presence of the restricted regions.

In real world location problems, situations are often encountered in which regions neither allow facility location nor travel through. Such regions are defined as barriers in the facility location literature. Examples of barriers would be impassable areas on the shop floor like machines, subassembly areas, input-output docks, etc. The available literature on location problems in the presence of barriers can be classified according to the following criteria: 
- shape of barriers (arbitrary, polygonal, circular, rectangular, etc.),

- size of new facility (infinitesimal or finite size),

- travel metric used (Euclidean, rectangular, etc.),

- type of objective (median or center).

In one the earliest facility location papers dealing with barriers, Katz and Cooper [8] investigated a problem with a circular barrier considering the median objective and the Euclidean distance metric. Larson and $\mathrm{Li}$ [9] developed an efficient algorithm for determining the shortest feasible rectangular path between two points in the presence of polygonal barriers. Discretization results for the $p$-median problem in the presence of arbitrarily shaped barriers under the rectangular distance metric were obtained by Larson and Sadiq [10]. The authors introduced a grid construction procedure that splits the feasible region into cells. By converting the original problem into an equivalent $p$-median problem on a network, the authors proved that an optimal set of facility locations can be chosen based solely on the grid points, i.e., the nodes (barrier vertices and/or demand points) and the points of intersection of any two node traversal lines.

Batta et al. [2] extended the results of [10] to include regions that prohibited facility location but allowed travel through. Aneja and Parlar [1] and Butt and Cavalier [3] developed heuristics for the 1 -median problem in the presence of polygonal barriers under the $l_{\mathrm{p}}$ distance metric. Though the center problem in $\mathfrak{R}^{2}$ without barriers has been extensively studied in the literature (e.g., books of Drezner [5], Love et al. [11] and Francis et al. [6]), very few references can be obtained for the corresponding problem in the presence of barriers. Minimax locations problems on a network have been studied in the book by Handler and Mirchandani [7]. p-center problems on a network have been extensively studied in the book by Mirchandani and Francis [12].

Nandikonda et al. [14] address the 1-center problem with arbitrarily shaped barriers under the rectangular distance metric. The authors divide the feasible region into cells as outlined by [10]. To overcome complications due to the center objective, they introduce a new concept to classify cells based on their cell corners. A solution procedure has been developed for each class of cells. The overall complexity of the solution procedure is shown to be polynomially bounded. Dearing et al. [4] obtain dominating set results for the 1-center location problem in the presence of convex polygonal barriers under the rectangular distance metric. The feasible region is decomposed into cells. A bisector algorithm, that finds bisectors by adapting an algorithm of Mitchell [13], outputs the set of dominated points. Based on the domination results, the authors develop a polynomial (in the number of demand points and number of extreme points of the polygonal barriers) time algorithm to solve the resulting nonconvex optimization problem. Their work differs from the work of Nandikonda et al. [14] in its solution approach as well as the shape of barriers.

In all the above-mentioned literature, the authors have assumed the new facility to be located as infinitesimal in size. However such an assumption is not always valid. Savas et al. [16] first considered the finite size "placement" problem of an arbitrarily shaped facility in the presence of arbitrary shaped barriers with the median objective and rectangular distance metric. By studying the behavior of the objective function, the authors identify the candidate(s) for optimal placement of a new facility with a fixed orientation and then for the same facility with a fixed server location. They also present a heuristic for the case when both facility orientation and server location are not known a priori. Our work differs from the work of Savas et al. [16] with respect to the nature of the objective. Also Nandikonda et al. [14] consider the same objective with an infinitesimal new facility whereas our work addresses the finite size facility placement problem.

In this paper, we consider the problem of "placing" a facility in the presence of barriers. This work can be practically applied to problems in layout analysis, where the objective, in many cases is to place a new department in the presence of existing departments (or to place a new machine in the presence of existing machines on the shop floor). An existing machine with its adjoining area comprising of space for associated equipment, input buffer, output buffer, scrap area can be considered to be a barrier.

The remainder of this paper is organized as follows. In Section 2, we describe and define our problem. In Section 3, we briefly revisit the grid construction procedure of [10] and the concept of "Equal Travel Time Lines" (ETTLs), as established in [2]. We then study and establish some new properties of ETTLs in Section 4. Section 5 considers the facility placement problem with a given orientation; this section is split into several sub-sections, each dealing with a different case of the problem. We analyze the complexity of our solution procedure in Section 6 for the fixed orientation problem. We 
present an example problem and its detailed analysis in Section 7 to elucidate our solution methodology. Conclusions and directions for future research are outlined in Section 8.

\section{Problem description}

Let there exist a finite number of barriers where travel through and facility placement are both prohibited. The existing users are distributed over a finite set of demand points located anywhere in the plane outside of or on the boundary of the barriers. A new facility is to be placed. The new facility communicates with the existing users through a single server located on its boundary. The 1-center facility placement problem is to determine the optimal placement of the facility such that the facility does not overlap with any of the existing barriers, and the maximum rectangular travel distance (circumventing barriers) from the server to any user is thereby minimized.

\subsection{Definitions and notations}

We assume that each barrier is a closed and bounded area in $\mathfrak{R}^{2}$, with finite area. Let $B_{j}$ (an open set) denote the set of points $(x, y) \in \mathfrak{R}^{2}$ contained strictly within barrier $j$. We also define $\overline{B_{j}}=$ $B_{j} \cup\{$ boundary of barrier $j\}$, to be a closed set. Each set $\overline{B_{j}}$ is called a barrier. Each barrier has a finite number of horizontal and vertical tangential lines. We let $B=\cup_{j} B_{j}$ and $\bar{B}=\cup_{j} \overline{B_{j}}$. Let $H$ (an open set) denote the set of points contained strictly within the new facility that is to be placed and let $\bar{H}=H \cup\{$ boundary of the facility $\}$. We note here that $B_{i} \cap B_{j}=\emptyset, i \neq j$ and $B_{j} \cap H=\emptyset$. The distinction between the inside and the boundary of a barrier/facility is necessary to allow travel on the boundary of the barrier/facility but not inside. Let $E(\bar{B})$ define the smallest rectangle (bounding rectangle) that encloses all barriers and users and whose sides are parallel to the $x$ - and $y$-axes.

For a finite size facility, the coordinates of a single point cannot convey full information about the placement of the facility in $\mathfrak{R}^{2}$. Hence, we let $l=[X, \alpha]$ denote the placement vector for the facility. Here, $X=(x, y)$ represents the location, i.e., point coordinates for the server. The angle $0 \leqslant \alpha<2 \pi$ between the $+x$-axis and the line joining the server location and a predetermined point $P$ on the facility (measured in the anti-clockwise direction) specifies the orientation of the facility. Fig. 1 shows the

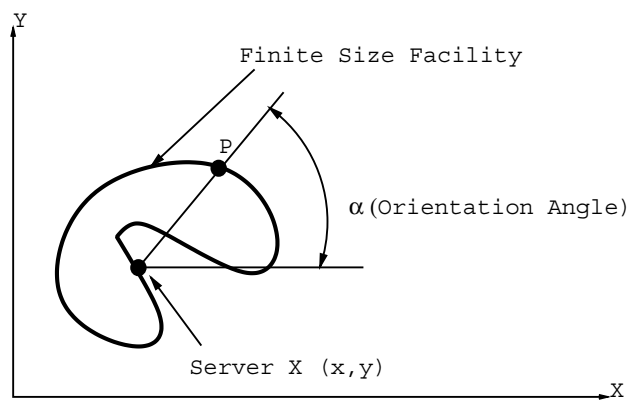

Fig. 1. A finite-size facility, its location and orientation.

placement vector. In summary, $X$ is the location and $\alpha$ is the orientation of the facility. Together they determine the placement of the facility.

We will now define the feasible region for the finite-size facility placement problem. Let $\bar{H}(X, \alpha)$ be the set of points that correspond to the facility when the server is at $X$ and has an orientation $\alpha$. $\bar{H}(X, \alpha)$ is a closed set. The feasible region is defined as follows:

$F=\{[X, \alpha]: H(X, \alpha) \cap B=\emptyset\}$.

We note here that $F$ may be composed of mutually disjoint sets.

\subsection{Problem definition}

There is interaction between users and the server. The extent of interaction between user $i$ and the server is denoted by $w_{i} \geqslant 0$. The interaction between any user and server takes place through a shortest feasible rectangular distance path that penetrates no barriers or facilities. Let $d_{l}(i, X)$ represent the length of such a shortest rectangular path between the user $i$ and server at $X$ when the facility placement is $l$. The subscript $l$ signifies that the distance is a function of the facility placement.

Let $D$ denote the set of all users. For a given facility placement $l=[X, \alpha]$, we define the function $J(l)$ as follows:

$J(l)=\max _{i \in D}\left\{w_{i} d_{l}(i, X)+\gamma_{i}\right\}$,

where $w_{i} d_{l}(i, X)$ is the weighted travel distance between user $i$ and the server $X$ of the new facility and $\gamma_{i}$ is the constant addendum associated with user $i$.

Then the weighted 1-center problem with arbitrarily shaped barriers can be denoted as follows:

$\min _{l \in F} J(l)$. 


\section{Background}

\subsection{Grid construction and cell formation}

A grid construction procedure in the presence of barriers that divides the feasible region $F$ into cells has been established in the work of Larson and Sadiq [10]. The same procedure is followed here. Let $Q\left(\overline{B_{j}}\right)$ denote the points of tangency of barrier $\overline{B_{j}}$, i.e., points on the boundary of $\overline{B_{j}}$ through which a horizontal and/or a vertical line can be passed and for which all points on the boundary sufficiently close to this point lie in or either on one side of the line or the other. The grid construction procedure is outlined as follows:

(i) Draw lines parallel to the $x$ - and $y$-axes through all barrier vertices and the users, with each line terminated at the first barrier interior encountered (i.e., point in $B$ ), else at $E(\bar{B})$, as illustrated in Fig. 2. (ii) Exclude from the set of lines in (i) any line extending from a barrier vertex that is not a user and where the vertex is the endpoint of the line. An example of the grid construction procedure is illustrated in Fig. 2. For further details of the grid construction procedure, we refer the interested reader to [10].

The resulting set of lines are called "node traversal lines" in [10]. For simplicity in presentation, let us refer to these lines as gridlines. We let $L_{\mathrm{h}}$ denote the set of horizontal gridlines and $L_{\mathrm{V}}$ denote the set of vertical gridlines. We define $L=L_{\mathrm{h}} \cup L_{\mathrm{v}}$ as the set of all gridlines. The barriers and $L$ divide $F$ into a number of cells, as illustrated in Fig. 2. Each cell boundary is composed of solely gridlines

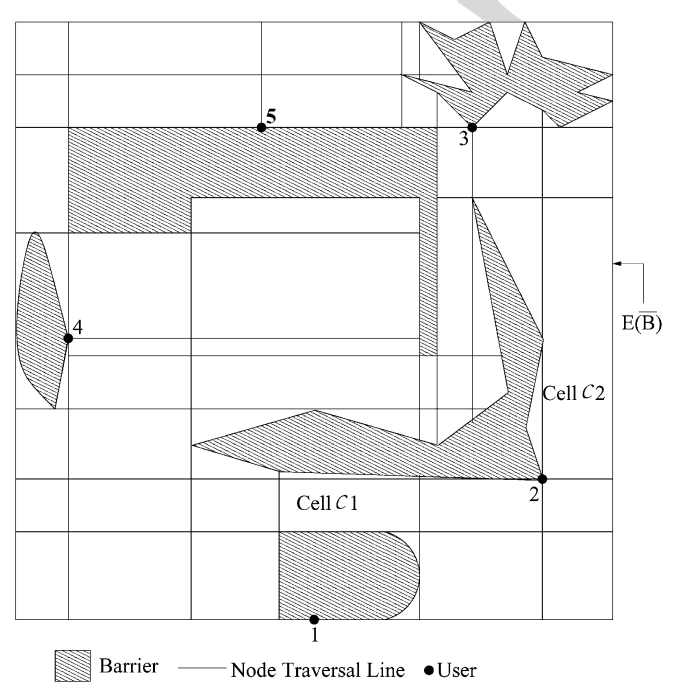

Fig. 2. Example of the grid construction procedure. or segments of barrier boundaries and gridlines. For a given cell $\mathcal{C}$, let us consider the points $\left(x_{\min }, y_{\min }\right),\left(x_{\max }, y_{\min }\right),\left(x_{\max }, y_{\max }\right),\left(x_{\min }, y_{\max }\right)$, where $x_{\min }, y_{\min }, x_{\max }, y_{\max }$ are the respective bounds on $x$ and $y$ in the cell. Clearly, at least one of the four points $\left(x_{\min }, y_{\min }\right),\left(x_{\max }, y_{\min }\right)$, $\left(x_{\max }, y_{\max }\right)$, or $\left(x_{\min }, y_{\max }\right)$ is contained in $\mathcal{C}$. All such points contained in $\mathcal{C}$, up to a maximum of four, are henceforth referred to as cell corners of $\mathcal{C}$. We will later utilize the following results, as proved in $[10,16]$.

Result 1 [10]. A shortest feasible rectangular path from a user to an infinitesimal point located in a cell $\mathcal{C}$ passes through a cell corner of $\mathcal{C}$.

Result 2 [10]. A shortest path in $\mathfrak{R}^{2}$ between any $\left(x_{a}, y_{a}\right)$ and $\left(x_{b}, y_{b}\right)$ can be found by restricting travel to nodal paths, i.e., paths containing a sequence of nodes $a-n_{1}-n_{2}-\cdots-n_{k}-b$ where $\left(a, n_{1}\right),\left(n_{1}\right.$, $\left.n_{2}\right), \ldots,\left(n_{k}, b\right)$ are pairs of simply communicating nodes (two nodes communicate simply if the presence of barriers causes no net increase in the travel distance between them).

Result 3 [16]. A nodal path between two points $\left(x_{a}, y_{a}\right)$ and $\left(x_{b}, y_{b}\right)$ can be represented as a "traversal line path" $P(a, b)$, i.e., a path that comes in contact with a sequence of horizontal and vertical gridlines, $h_{1}, h_{2}, \ldots, h_{m}$ and $v_{1}, v_{2}, \ldots, v_{n}$.

Cells that do not share a common boundary with a barrier $\bar{B}$ are rectangular and have four cell corners. Cells that share their boundaries with a barrier may not be rectangular and hence may have less than four cell corners. Let the subset $\zeta^{p}$ denote all cells with $p$ cell corners. Based on the previous discussion, we denote by $\zeta$ a set of all cells, then $\zeta=\zeta^{1} \cup \zeta^{2} \cup \zeta^{3} \cup \zeta^{4}$.

\subsection{Equal Travel Time Lines and subcells}

For a fixed location $X \in \mathcal{C}$, we can meaningfully talk of the assignment of users to cell corners of $\mathcal{C}$. If the assignment of users to cell corners does not change upon moving the location $X$ in $\mathcal{C}$, the distance functions are linear and monotonous over the cell $\mathcal{C}$. However, any change in the assignment of users to cell corners necessitates the construction of "Equal Travel Time Lines" (ETTL). The concept of ETTLs was introduced in the work of Batta et al. [2] and subsequently utilized in the work of 


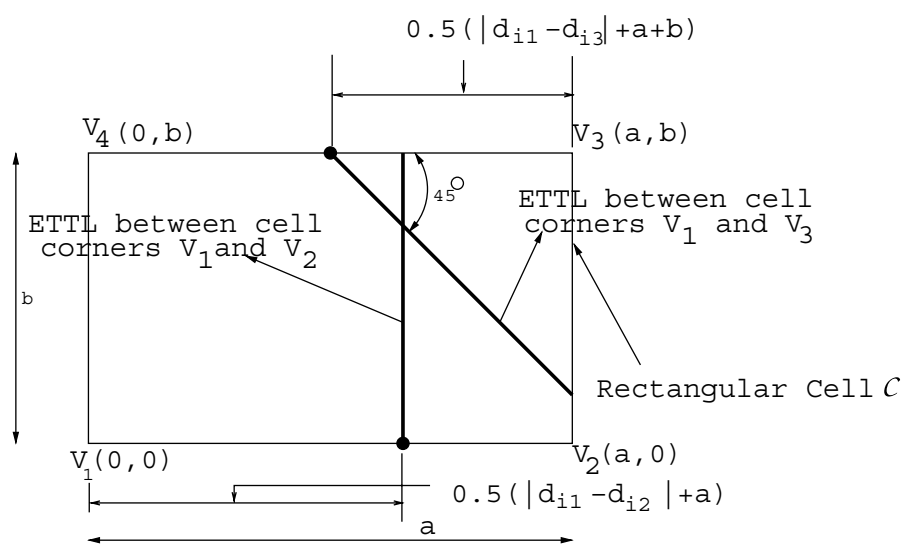

Fig. 3. Example of an ETTLs in a rectangular cell.

Nandikonda et al. [14]. Details regarding construction of ETTLs can be found in $[2,14]$.

Let us consider a cell $\mathcal{C}$ with corners $V_{k}$, $k=1,2,3,4$ as shown in Fig. 3. For the sake of simplicity and without loss of generality, let the corners $V_{1}, V_{2}, V_{3}, V_{4}$ have coordinates $(0,0),(a, 0),(a, b)$, $(0, b)$, respectively. Let the shortest rectangular distance from user $i$ to cell corner $V_{k}$ be denoted by $d_{i k}$ for $k=1,2,3,4$. If $\left|d_{i 1}-d_{i 2}\right|<a$, where $a$ is the length of the edge $\overline{V_{1} V_{2}}$, then an ETTL with respect to $i$ between corners $V_{1}$ and $V_{2}$ is generated such that it is perpendicular to the edge $\overline{V_{1} V_{2}}$ at a distance $\frac{1}{2}\left\{\left|d_{i 1}-d_{i 2}\right|+a\right\}$ from the cell corner that is closest to user $i$. The cases between other adjacent cell corners can be dealt with similarly. If $\left|d_{i 1}-d_{i 3}\right|<a+b$, where $b$ is the length of the edge $\overline{V_{2} V_{3}}$, then an ETTL with respect to $i$ between corners $V_{1}$ and $V_{3}$ is generated that touches the cell boundary at a distance $\frac{1}{2}\left\{\left|d_{i 1}-d_{i 3}\right|+a+b\right\}$ from the cell corner that is closest to user $i$. It is pertinent to mention here that ETTLs generated due to diagonally opposite cell corners are inclined at $45^{\circ}$ to the edges of a 4-cornered cell, e.g., the ETTL generated due to $V_{1}$ and $V_{3}$ makes an angle of $45^{\circ}$ with the edges $\overline{V_{2} V_{3}}$ and $\overline{V_{3} V_{4}}$. After the construction of ETTLs, which are induced on a cell $\mathcal{C}$ by user $i$, has been completed, the same procedure is repeated for all other users $k, k \neq i$. ETTLs partition a cell $\mathcal{C}$ into subcells $S C$. All ETTLs generated by the users in Fig. 2 are illustrated in Fig. 4. For example, con-

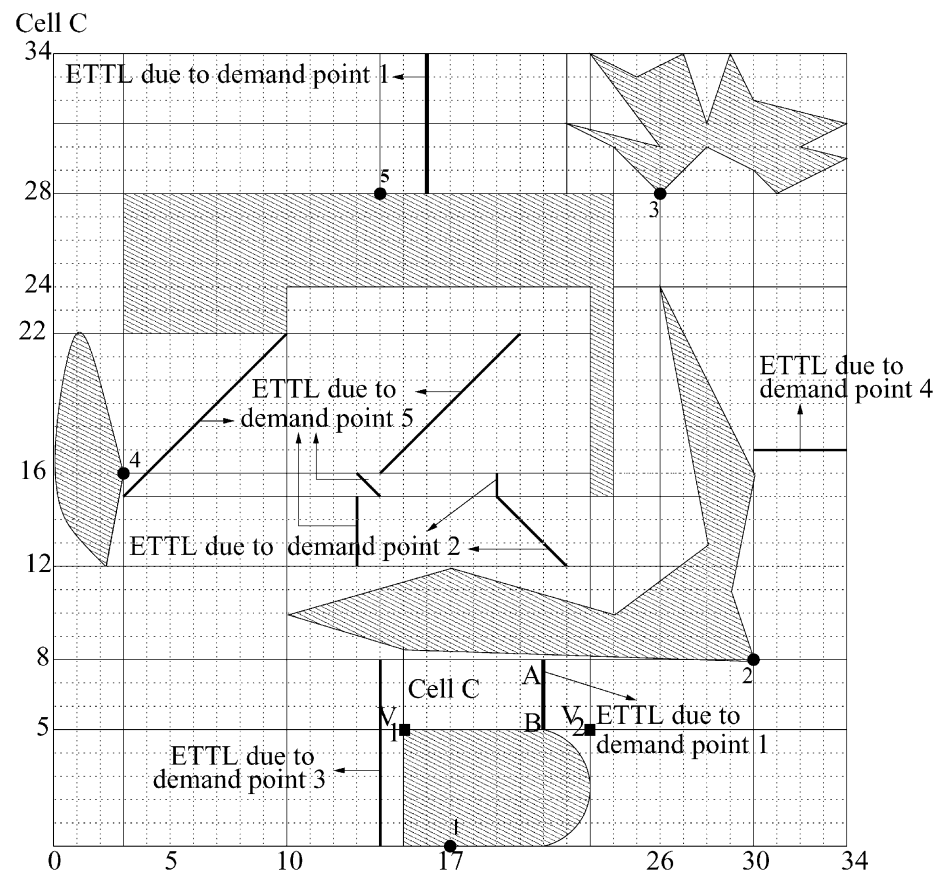

Fig. 4. Cells and subcells. 
sider ETTL $A B$ in cell $\mathcal{C}$ in Fig. $4 . A B$ is an ETTL in the following sense: if $X$ is located at a point on $A B$, a shortest rectangular path between $X$ and user 1 goes through either cell corner $V_{1}$ or $V_{2}$ (in other words, one has to cover equal distance (hence the name ETTL) to travel from user 1 to $X$, either through $V_{1}$ or $V_{2}$ ). Clearly ETTL $A B$ helps to uniquely assign user 1 to cell corner $V_{1}$ or $V_{2}$ depending on the location of $X \in \mathcal{C}$.

It is pertinent to mention here the following result proved in [14].

Result 4. A demand point generates at most one ETTL in a 4-cornered cell.

\section{Additional properties of ETTLs}

\subsection{Existing ETTLs may move}

As discussed in Section 3.2, an ETTL may be generated between any pair of cell corners of a 4-cornered cell. Due to the finite size of the new facility, an existing ETTL may move. In fact, the following are now possible.

1. Due to its finite size and shape, the length of the shortest rectangular path between a user $i$ and the new facility's server $X, d(i, X)$, may increase. Consider the ETTL generated in the cell $\mathcal{C}$ between adjacent cell corners $V_{1}$ and $V_{2}$, as illustrated in Fig. 5a. Due to placement of the new facility as illustrated in Fig. 5b, $d(i, X)$ increases by $2 p$ (note that user $i$ is uniquely assigned to cell corner $V_{1}$ ). Hence the ETTL moves closer to $V_{1}$ by $p$. We conclude that the position of an existing ETTL may thus move when a finite sized facility is fully contained in a cell. Similar analysis can be performed for ETTLs between other pairs of adjacent as well as opposing cell corners.

2. The finite sized new facility may block cell corners of cell $\mathcal{C}$ through which a user $i$ communicates with $X \in \mathcal{C}$. Consequently $d(i, X)$ may increase and an existing ETTL in $\mathcal{C}$ may move. However unlike the previous case 1, the new facility now intersects gridlines. The amount of movement of the ETTL is a function of the increment of $d(i, X)$. We refer the interested reader for more details to [15].

Our solution methodology proposed later is based on a cell decomposition approach. When the new finite size facility is not fully contained in a cell, i.e., it intersects gridlines (Section 5.2.2), the shortest rectangular path between users and the server of the new facility is affected. Consequently the unique assignment of users to cell corners may change. Accurate distance measurements between users and the server is central to our solution methodology. Hence it is crucial to consider the movement of ETTLs. Note that this complexity is not encountered in [14] due to the infinitesimal size of the new facility.

\subsection{ETTL intersections in a cell}

We conclude this section with the following lemma, which helps to simplify our analysis.

Lemma 4.2.1. ETTLs between both pairs of opposing cell corners in a 4-cornered cell cannot exist.
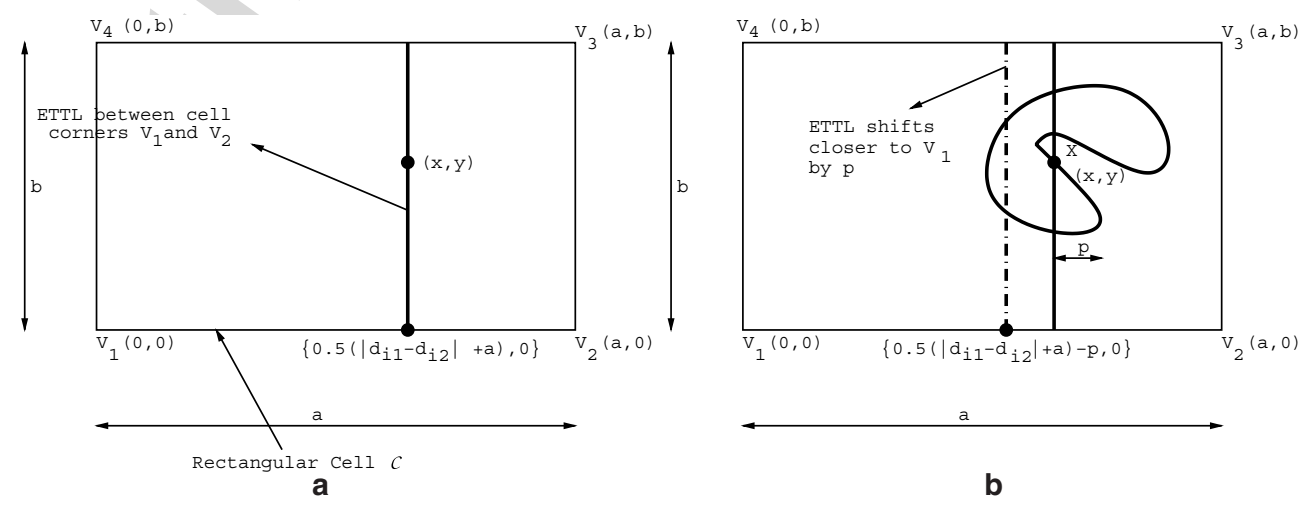

Fig. 5. Movement of ETTLs. 


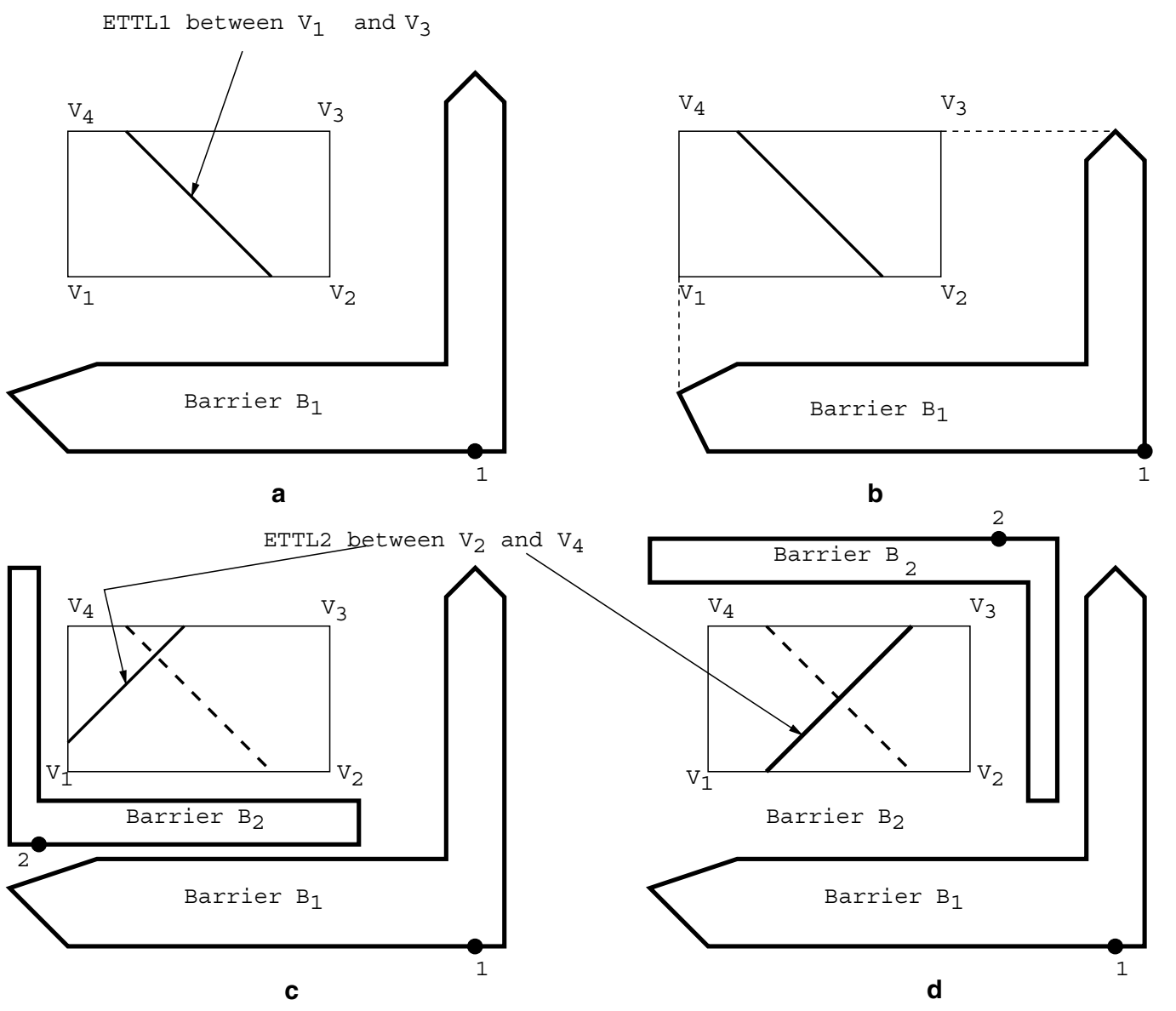

Fig. 6. Figure for Lemma 4.2.1.

Proof. Consider a 4-cornered cell $\mathcal{C}$ with cell corners $V_{1}, V_{2}, V_{3}, V_{4}$. Assume that an ETTL, labeled ETTL1 exists between $V_{1}$ and $V_{3}$ due to user 1 associated with barrier $B_{1}$, as illustrated in Fig. 6a. This happens when $V_{2}$ is not rectangularly visible to 1 . (Otherwise an ETTL would not exist between $V_{1}$ and $V_{3}$ and an ETTL could exist between $V_{2}$ and $V_{3}$ or $V_{1}$ and $V_{2}$.) However $V_{1}$ and $V_{3}$ may be rectangularly visible to 1 if the cell is formed due to barrier traversal lines associated with $B_{1}$, as illustrated in Fig. 6b.

Let us also assume that user 2, associated with barrier $B_{2}$ generates an ETTL, labeled ETTL2 in $C$ between $V_{2}$ and $V_{4}$, as shown in Fig. $6 \mathrm{c}$ and $\mathrm{d}$. Depending on the placement of $B_{2}, V_{1}$ and $V_{3}$ (as in Fig. 6c and d) will not be rectangularly visible to 2 . In either case, a shortest rectangular path from server location 1 to $V_{1}$ (in Fig. 6c) and to $V_{3}$ (in Fig. 6d) must pass through cell corner $V_{2}$. Hence an ETTL would exist between (adjacent cell corners) $V_{2}$ and $V_{3}$ in the case depicted in Fig. 6c and between (adjacent cell corners) $V_{1}$ and $V_{2}$ in the case depicted Fig. 6d, not between (opposite) cell corners $V_{1}$ and $V_{3}$. This contradicts our first assumption. The lemma follows.

Lemma 4.2.1 helps to restrict the number of subcells generated, as evident later in solution complexity analysis in Section 6. Based on the previous background, we now move on to the actual solution methodology of our problem.

\section{Solution procedure}

\subsection{Introduction}

Before a new facility is placed, the gridlines that are drawn due to the existing barriers and users were adequate to provide the framework that is required to obtain the minimum feasible rectangular distance path between any two users. Placement of a new facility alters the existing framework. Specifically, two things happen. Firstly, the facility itself acts as a barrier to travel. Hence a new set of 
traversal lines parallel to the coordinate axes must be passed through the vertices of the facility and the server on the facility as outlined in [10]. Ref. [16] has referred to these lines as facility traversal lines. Secondly, some existing gridlines will be terminated as they will encounter the interior of the new facility. As a result of placing a new facility, a new grid structure will be obtained.

Savas et al. [16] consider the 1-median problem of placing a finite-sized facility. The authors take advantage of the concave property of the distance functions and the nature of the objective function which is a positive linear combination of concave functions. However the objective (minimax) function of our problem does not help in deducing any such property. Hence it becomes imperative in our problem to represent the distance functions analytically, as shown in later sections. This becomes challenging especially because of the arbitrary shape of the barriers and the new facility to be placed. Some concepts of Nandikonda et al. [14] are useful here as their work deals with location of an infinitesimal facility under the center objective. However since our problem addresses the finite size facility placement problem, we cannot directly extend the results of [14]. Analogous to [14], we split up our analysis into different cases. Unlike [14] due to the finite size of the facility to be placed, we have to identify a feasible region of location in each case. Also, in our problem, if the new facility to be located moves, the assignment of users to cell corners may change (as described in Section 4).

To facilitate our analysis, we conclude this section by defining the smallest enclosing rectangle of the new facility as $E(\bar{H})$. Similar to what we have previously defined for a cell, let the corners of $E(\bar{H})$ be denoted as $H_{k}, k=1,2,3,4$. We note that as the facility orientation remains fixed, so does $E(\bar{H})$.

\subsection{Optimal placement candidates for the facility with fixed orientation}

In this section, we deal with the placement of a facility with known orientation $\alpha_{0}$. We split up our analysis into two cases:

- when the facility placement does not cut off any element of the set $L$,

- when the facility placement cuts off at least one element of the set $L$.

\subsubsection{The facility placement does not cut off any element of $L$}

We note at the outset that we are concerned with the location of the server such that the new facility does not cut off any element of the set $L$, i.e., the facility is fully contained within a cell $\mathcal{C}$. This means that the set of feasible locations for the server $\mathcal{F} \subseteq \mathcal{C} . \mathcal{F} \subseteq \mathcal{C}$ can be obtained by moving the facility inside the cell such that it does not cut off any element of $L$. Based on the previous background, we now consider the following cases:

\section{Case 1: The facility is fully contained in a 1-cornered cell}

A 1-cornered cell $\mathcal{C}$ can be generated in four ways by choosing any one of the four possible cell corners $\left(x_{\min }, y_{\min }\right),\left(x_{\max }, y_{\min }\right),\left(x_{\max }, y_{\max }\right),\left(x_{\min }, y_{\max }\right)$. Let us consider the new facility to be contained fully inside $\mathcal{C}$ with cell corner $V_{1}$, as illustrated in Fig. 7 . The following lemma establishes the fact that in such a scenario, the facility can be moved inside the cell thereby bringing the server closer to $V_{1}$.

Lemma 5.2.1. Consider the new facility to be fully contained in a 1-cornered cell. Its optimal location is either (i) a unique point lying inside the cell, or (ii) on the vertical or horizontal tangent passing through the only cell corner, or (iii) the cell corner itself.

Proof. Let $X$ be the optimal server location such that it is different from locations (i), (ii), and (iii) in the lemma statement. If the facility is moved from $X$ to $X_{1}$ along the rectangular path $X O X_{1}$ (such that $\angle X O X_{1}=90^{\circ}$ as illustrated in Fig. 7) until at least one facility traversal line coincides with the tangent (horizontal or vertical depending on the shape of the 1-cornered cell) passing through $V_{1}$, the distance between the new server location $X_{1}$ and all users necessarily decreases. This follows from Result 1 [10] mentioned earlier and hence a contradiction is established. The optimal facility location (i) is thus obtained. The optimal location mentioned in (ii) may be obtained if $X$ lies on $E(\bar{H})$. Location (iii) is a special case of locations (ii).

Since similar arguments can be made for any 1-cornered cell formed by choosing any one of the four points $\left(x_{\min }, y_{\min }\right),\left(x_{\max }, y_{\min }\right),\left(x_{\max }, y_{\max }\right)$, $\left(x_{\min }, y_{\max }\right)$, the lemma follows.

Note that the optimal location of the new facility (for the fixed orientation problem) is given by the optimal location of $X$, its server. Clearly, the optimal server location for the case illustrated in 


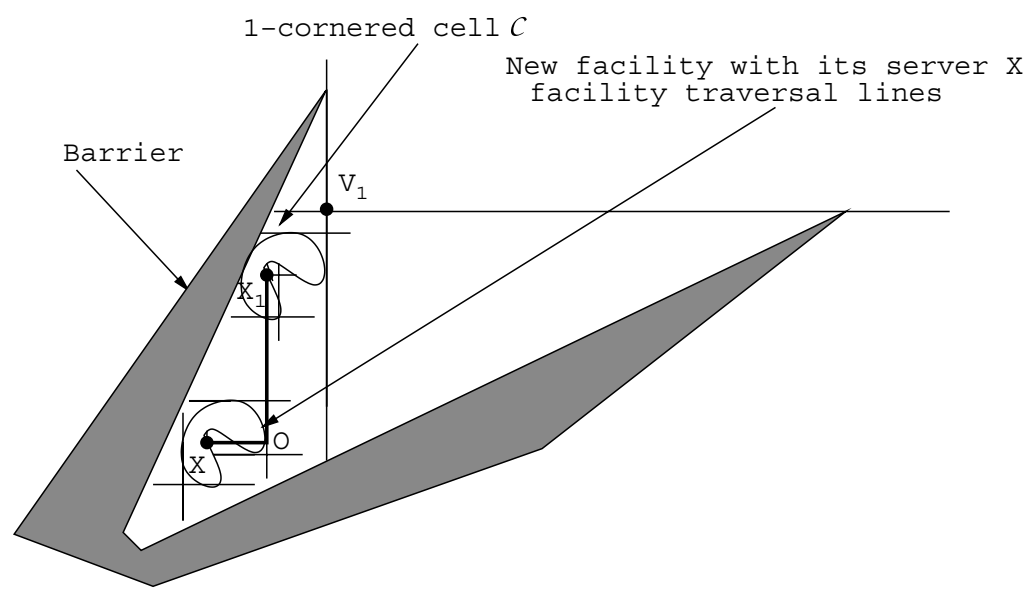

Fig. 7. A 1-cornered cell (used in the proof of Lemma 5.2.1).

Fig. 7 is $X_{1}$. From Lemma 5.2.1, in a cell $\mathcal{C} \in \zeta^{1}$, the point $X_{1}$ dominates all other points. Hence it is sufficient to evaluate the 1-center objective function at $X_{1}$.

Case 2: The facility is fully contained in a 2-cornered cell

A 2-cornered cell $\mathcal{C}$ can be generated by choosing any two of the four possible cell corners $\left(x_{\min }, y_{\min }\right)$, $\left(x_{\max }, y_{\min }\right),\left(x_{\max }, y_{\max }\right),\left(x_{\min }, y_{\max }\right)$. This can be done in six ways. Fig. 8a illustrates a 2-cornered cell with the cell corners $V_{1}\left(x_{\min }, y_{\min }\right), V_{2}\left(x_{\max }, y_{\min }\right)$ while Fig. $8 \mathrm{~b}$ illustrates the case with $V_{1}\left(x_{\max }, y_{\min }\right)$, $V_{2}\left(x_{\min }, y_{\max }\right)$. We now state and prove the following lemma.
Lemma 5.2.2. Consider the new facility to be fully contained in a 2-cornered cell. Its optimal location is such that at least one edge of the facility bounding rectangle $E(\bar{H})$ coincides fully or partially with one of the shortest rectangular paths (SRP) between the two cell corners.

Proof. By definition, a cell corner must be either $\left(x_{\min }, y_{\min }\right),\left(x_{\max }, y_{\min }\right),\left(x_{\min }, y_{\max }\right)$, or, $\left(x_{\max }, y_{\max }\right)$. Hence six cases can be generated by choosing any two of the four aforementioned points as cell corners.

Let $X$ be the optimal server location such that no edge of $E(\bar{H})$ coincides with the SRP between

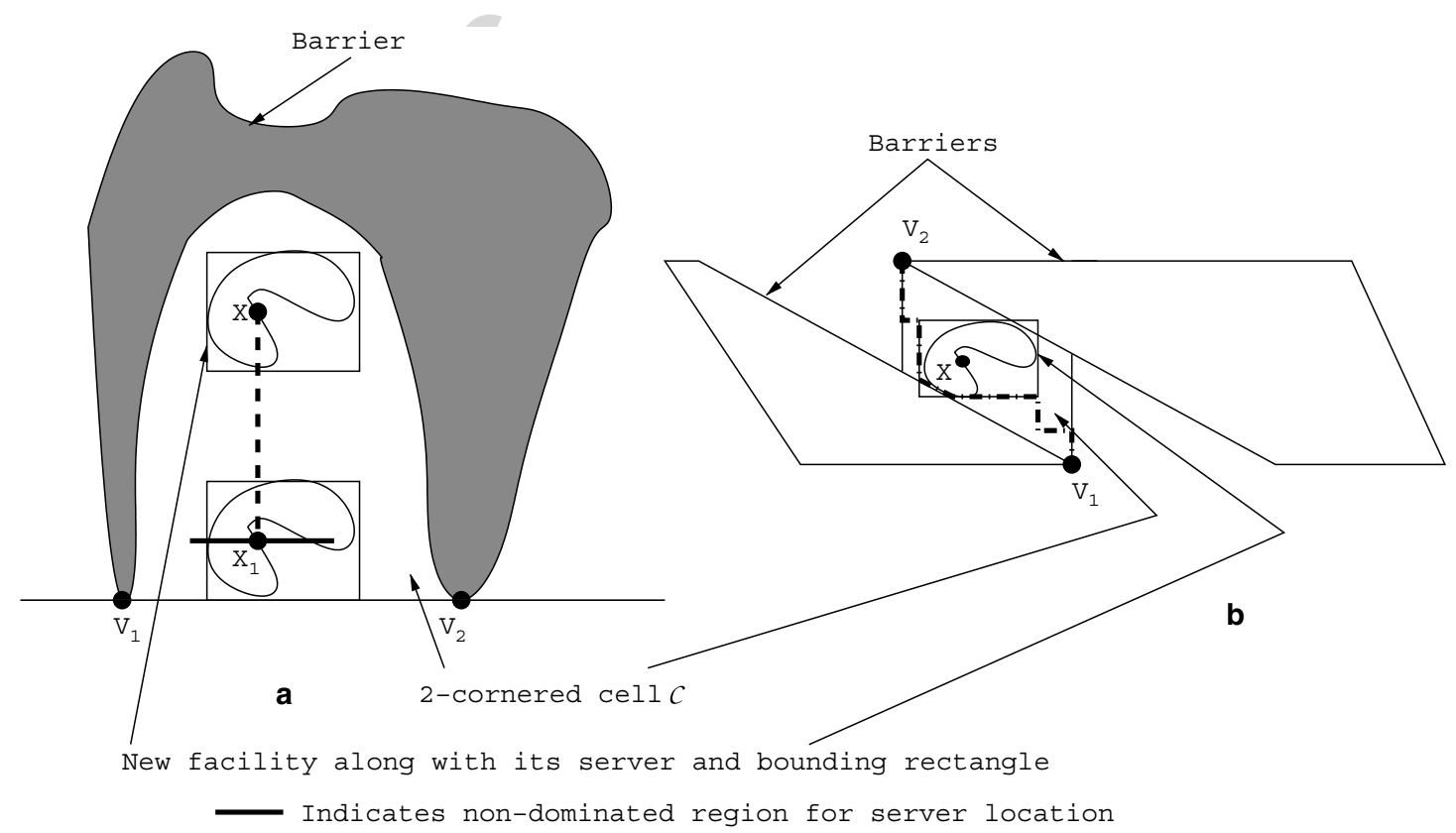

Fig. 8. A 2-cornered cell (used in the proof of Lemma 5.2.2). 
$V_{1}$ and $V_{2}$. However, as illustrated in Fig. 8a (2cornered cell with $\left(x_{\min }, y_{\min }\right)$ and $\left(x_{\max }, y_{\min }\right)$ as cell corners), we can move the facility along the perpendicular $X X_{1}$ until $E(\bar{H})$ coincides fully with the SRP between $V_{1}$ and $V_{2}$. Since the rectangular distance between $X_{1}$ and all users necessarily decreases, a contradiction is established.

For the case depicted in Fig. 8b, the size of the facility $\bar{H}$ is such that $E(\bar{H}) \cap B_{j} \neq \emptyset$. However as demonstrated in Fig. 8b, a SRP can always be drawn between $V_{1}$ and $V_{2}$ that partially coincides with edges of $E(\bar{H})$. The conditions of the lemma are thus met. Similar arguments hold for other possible configurations of a 2-cornered cell. The lemma follows.

The immediate implication of Lemma 5.2.2 is the following: for the case depicted in Fig. 8a (i.e., when a 2-cornered cell is formed between adjacent cell corners), the "nondominated" region $\mathcal{D}$ is a line segment. Any point on this line $\mathcal{D}$ dominates any feasible $(x, y) \in \mathcal{C}$ such that $(x, y) \notin \mathcal{D}$. This line segment can be obtained by moving the facility inside the cell $\mathcal{C}$, such that $B_{j} \cap H=\emptyset$ and $E(\bar{H})$ coincides with the line segment joining $V_{1}$ and $V_{2}$. D is demonstrated in Fig. 8 by a bold line.

For the case depicted in Fig. 8b, (i.e., a 2-cornered cell with opposing cell corners), the server $X$ can be moved along a line (joining $X$ and some point on the facility's boundary) that makes an angle of $45^{\circ}$ with the $+x$-axis without altering the minimum rectangular distance between a user $i$ (constrained to pass through $V_{1}$ or $V_{2}$ ) and $X$. (The idea is similar to the case of 3-cornered cell and is discussed in detail in the following case.) The nondominated region $\mathcal{D}$ can be obtained by moving the facility inside $\mathcal{C}$ such that $B_{j} \cap H=\emptyset$ (in a similar manner as discussed next for 3-cornered cells).

Based on the previous background, we now proceed to determine the optimal server location. Clearly cell corners $V_{j}$ and corners $H_{k}$ of $E(\bar{H})$ are rectangularly visible for $j=k=1,2$. Hence the distance between user $i$ and the facility server $X$ can be expressed as follows:

$d(i, X)=d\left(i, V_{j}\right)+d\left(V_{j}, H_{j}\right)+d\left(H_{j}, X\right)$,

where $d\left(i, V_{j}\right)$ and $d\left(H_{j}, X\right)$ are constants while $d\left(V_{j}, H_{j}\right)$ is a linear function of $x$ and $y$.

The 1-center facility placement problem in a 2-cornered cell $\mathcal{C}$ is as follows:

$\min _{X \in \mathcal{D}} \max _{i \in D}\left[w_{i} d(i, X)+\gamma_{i}\right]$.

The 1-center facility placement problem can thus be solved by performing a line search over the nondominated segment $S P \in \mathcal{D}$.

Case 3: The facility is fully contained in a 3-cornered cell

Following the discussion in Case 2, four possible cases are generated when any three of the four cell corners $\left(x_{\min }, y_{\min }\right),\left(x_{\max }, y_{\min }\right),\left(x_{\max }, y_{\max }\right)$, $\left(x_{\min }, y_{\max }\right)$ are selected to represent a 3-cornered cell. Fig. 9 shows a 3 -cornered cell with the cell

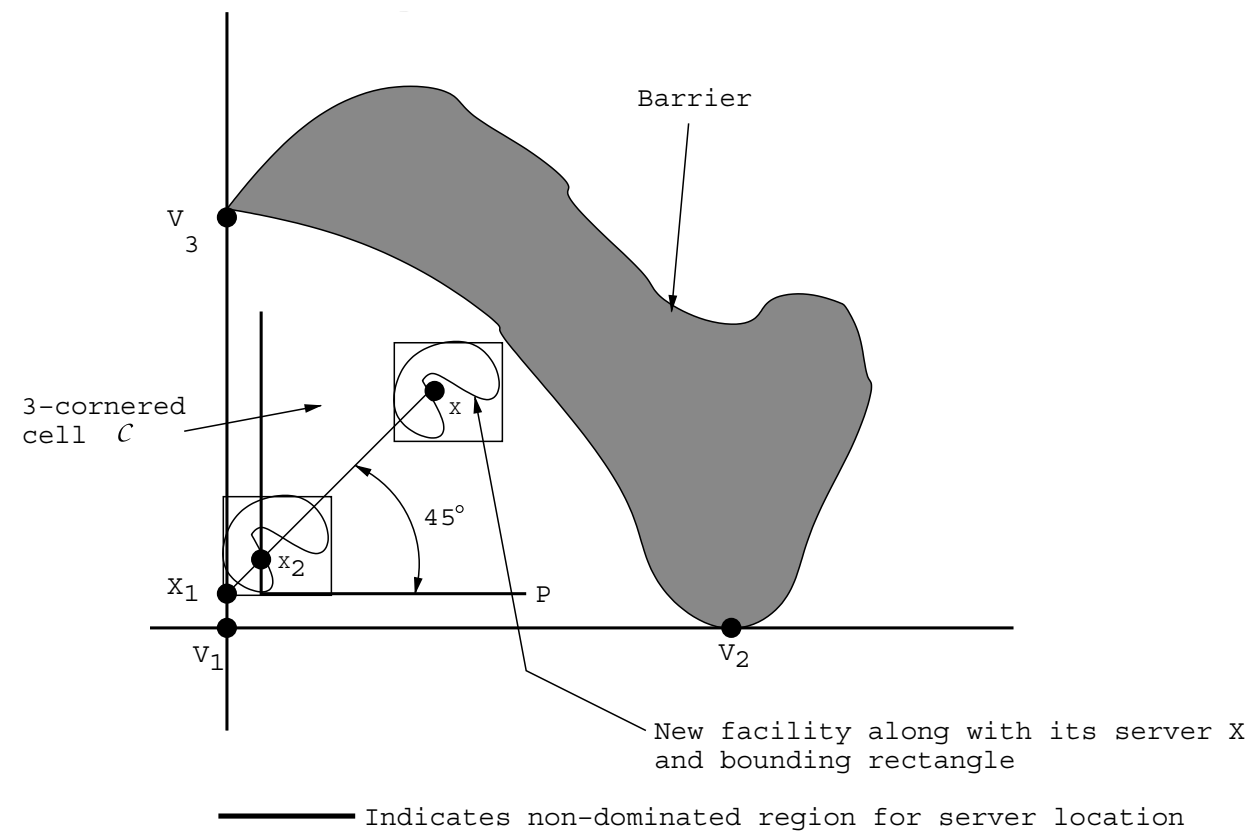

Fig. 9. A 3-cornered cell (used in the proof of Lemma 5.2.3). 
corners $V_{1}\left(x_{\min }, y_{\min }\right), V_{2}\left(x_{\max }, y_{\min }\right)$ and $V_{3}\left(x_{\min }\right.$, $\left.y_{\max }\right)$. We now state and prove the following lemma.

Lemma 5.2.3. Consider the new facility to be fully contained in a 3-cornered cell. Its optimal location is such that at least one edge of the facility bounding rectangle $E(\bar{H})$ coincides with any edge of the cell that is not common to a barrier boundary.

Proof. Let us consider the new facility to be contained fully inside a 3-cornered cell $\mathcal{C}$ with cell corners $V_{1}, V_{2}, V_{3}$, as illustrated in Fig. 9. Let us consider the point $X_{1}$ on the edge $V_{1} V_{3}$ such that $\angle X X_{1} P=45^{\circ}$. By moving the server $X$ of the new facility to $X_{2}$ along $X X_{1}$, the minimum rectangular distances from any user $i$ (constrained to pass through $V_{2}$ or $V_{3}$ ) to the server $X$ remains unaltered. Since we are considering the rectangular distance metric, any increase in $y$-distance from $X$ to $V_{2}$ or $V_{3}$ is compensated by a decrease in $x$-distance and vice versa. However for any user $i$ constrained to pass through $V_{1}$, the minimum rectangular distance between $i$ and $X$ strictly decreases. Similar arguments apply if $X_{1}$ lies on $V_{1} V_{2}$. Since we assume that the facility is fully contained inside the cell, the lemma follows.

Other cases of 3-cornered cells can be dealt with in a similar manner. An immediate implication of Lemma 5.2.3 is the following: the nondominated region $\mathcal{D}$, when the new facility is fully contained in a 3-cornered cell, is composed of line segments that are parallel to the edges of the cell that are not common to a barrier boundary. $\mathcal{D}$ can be obtained by moving the facility inside $\mathcal{C}$ such that $B_{j} \cap H=\emptyset . \mathcal{D}$ is demonstrated in Fig. 9 by bold lines.

Based on the previous background, the problem to determine the optimal server location over a segment $\mathcal{D}$ (when the new facility is fully contained in a 3-cornered cell), can be solved by performing a line search alike Case 2.

Before moving on to case 4 , we observe that Lemmas 5.2.1, 5.2.2 and 5.2.3 extend the results obtained in Theorem 1 of Nandikonda et al. [14] to account for finite size of the new facility in our work.

\section{Case 4: The facility is fully contained in a} 4-cornered cell

A 4-cornered cell $\mathcal{C}$ may be subdivided into subcells $S C$ by ETTLs between adjacent and/or opposing cell corners. Each subcell $S C$ is defined by a set

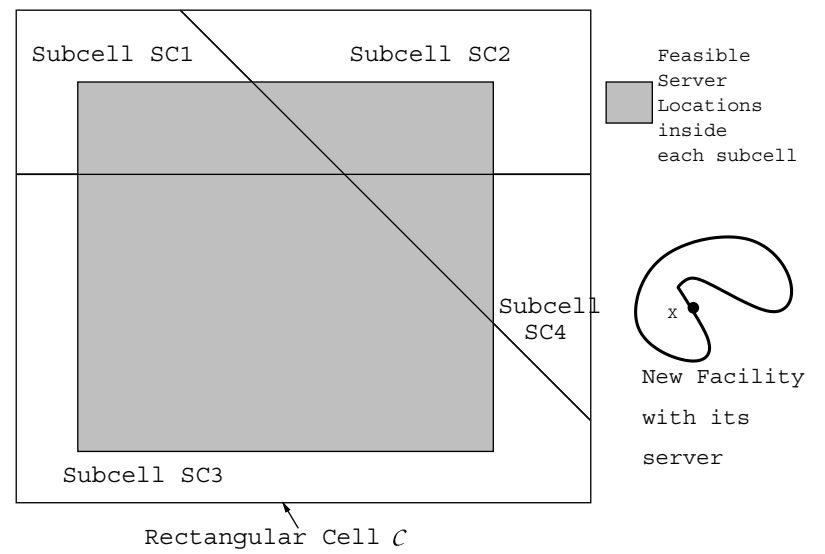

Fig. 10. Figure for Lemma 5.2.4.

of linear line segments (ETTLs) and the boundary of a rectangular cell $\mathcal{C}$ to which $S C$ belongs. It is straightforward to see that any $S C$ is a convex polyhedron (for discussion, see Fig. 10). Let $\mathcal{F}$ denote a feasible region within a $S C$ where a server of the new facility can be located. Note, however, that the new facility itself may intersect ETTLs and may cross over into one or more neighboring subcells. One can show the following result Lemma 5.2.4.

Lemma 5.2.4. $\mathcal{F} \subseteq S C$ are convex polyhedra.

Proof. Let us consider any subcell $S C$ of a rectangular cell $\mathcal{C}$, as shown in Fig. 10. $\mathcal{F} \subseteq S C$ is obtained by moving the new facility along the boundary of $S C$. Thus the server $X$ moves parallel to the edges of $S C$. The lemma follows since each subcell $S C$ is a convex polyhedron.

Hence $\mathcal{F} \subseteq S C$ can be represented by a set of inequalities

$A^{\mathcal{F}}\left(\begin{array}{l}x \\ y\end{array}\right) \leqslant b^{\mathcal{F}}$.

The rectangular distance from a user $i$ to the server $X$ can be expressed as follows:

$d(i, X)=d\left(i, V_{j}\right)+d\left(V_{j}, H_{k}\right)+d\left(H_{k}, X\right)$,

where $d\left(i, V_{j}\right)$ and $d\left(H_{k}, X\right)$ are constants while $d\left(V_{j}, H_{k}\right)$ is a linear function of the coordinates of the server, i.e., $x$ and $y$. Note that a pair of $V_{j}, H_{k}$ are rectangularly visible if $j=k$. Hence a user $i$ assigned to cell corner $V_{j}$ will communicate with the server $X$ through corner $H_{j}$ of the facility bounding rectangle. Since $H_{k}, k=1,2,3,4$ and $X$ may not be rectangularly visible, Results 2 and 3 mentioned in 
Section 3.1 can be utilized to evaluate the distance function $d\left(H_{k}, X\right)$.

The 1-center facility placement problem over each $\mathcal{F} \subseteq S C \subseteq \mathcal{C}$ can be formulated as a linear program as follows:

\section{minimize $Z$,}

subject to $w_{i}\left[d\left(i, V_{j}\right)+d\left(V_{j}, H_{j}\right)+d\left(H_{j}, X\right)\right]+\gamma_{i} \leqslant Z$

$$
\begin{aligned}
& \text { for } i \in \mathcal{V}_{k}, \forall k, \\
& A^{\mathcal{F}}\left(\begin{array}{l}
x \\
y
\end{array}\right) \leqslant b^{\mathcal{F}} .
\end{aligned}
$$

$\mathcal{V}_{k}$ denotes the set of users uniquely assigned to cell corner $V_{k}$, such that $\cup_{k} \mathcal{V}_{k}=D$. Solution to the linear program gives the optimal 1-center location in $\mathcal{F}$.

\subsubsection{The facility placement cuts off at least one element of $L$}

When the new facility is placed over a number of gridlines, the facility may interfere with the shortest rectangular paths between the existing users. The consequence of the facility cutting off a number of gridlines is that the shortest feasible rectangular path from a user $i$ to the server location $X$ may have to travel around the facility, i.e., the facility itself may act as a barrier to travel between the user and its server. Hence ETTLs may move, as discussed earlier in Section 4.1. Consequently the assignment of users to cell corners may change. The placement of the new facility determines the cell corner through which a user $i$ communicates with the server $X$ of the new facility.

Let us consider a feasible initial placement $l$ of the facility such that it cuts off at least one element of $L$. We assume here that no element of $L$ coincides with any facility traversal line. Following the concept of Savas et al. [16], we identify the set of placements $\mathcal{Q}$ (of the server $X$ ) such that when $l \in \mathcal{Q}$, the facility will always interfere with the same element(s) of $L$. The idea is elucidated in Fig. 11a, in which the new facility cuts off two gridlines, marked as Line $A$ and Line $B$. The set $\mathcal{Q}$ obtained by moving the facility such that it interferes with no gridline other than Line $A$ and Line $B$ is also shown. It is pertinent to note the following here:

1. $\mathcal{Q}$ may be composed of mutually disjoint sets, as illustrated by Fig. $11 \mathrm{~b}$.

2. $\mathcal{Q}$ may be nonconvex nonpolygonal, as illustrated by Fig. 12a.

3. $\mathcal{Q}$ may be convex polygonal, as illustrated by Fig. 11a.

4. $\mathcal{Q}$ may be nonconvex polygonal, as illustrated by Fig. $12 b$.

To demonstrate the existence of the previous cases, we have considered a rectangular new facility in some cases for the sake of simplicity.

The 1-center problem is solved over each $\mathcal{Q} \cap \mathcal{C}$, since $\mathcal{Q}$ may span more than one cell $\mathcal{C}$. For the $\mathcal{Q} \cap \mathcal{C}$ under consideration, the shortest rectangular

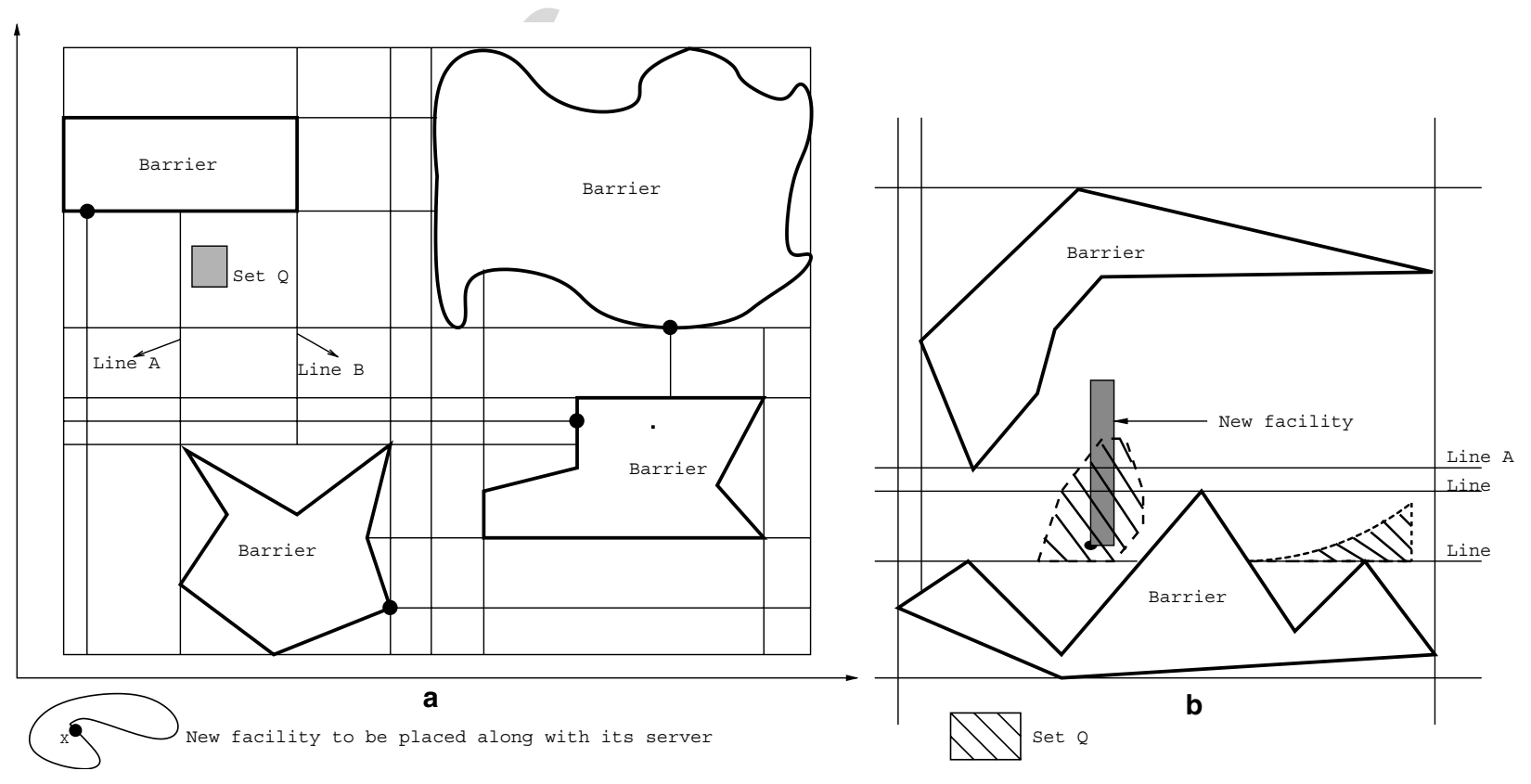

Fig. 11. Illustration for the set $\mathcal{Q}$. 

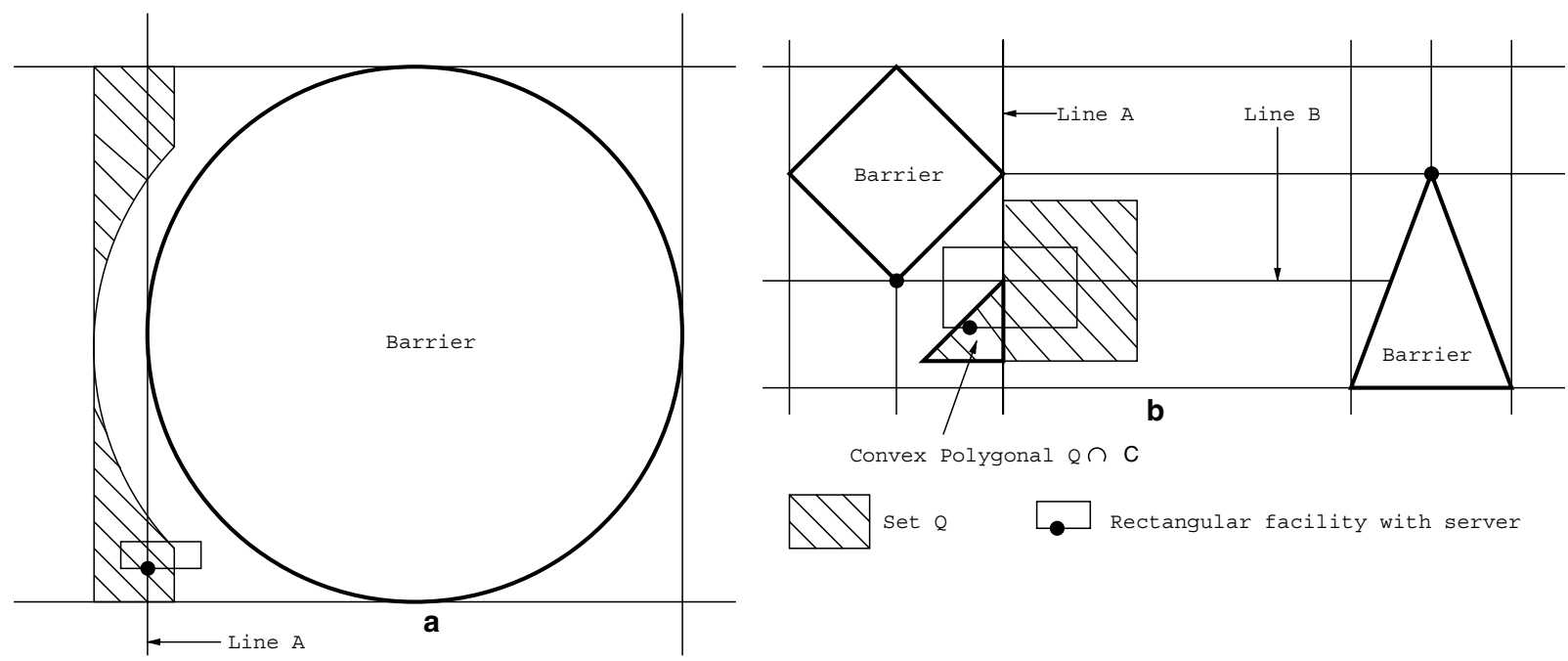

Fig. 12. Illustration for different shapes of $\mathcal{Q}$.

path from a user $i$ to $X$ is constrained to pass through the cell corners of $\mathcal{C}$. Also as illustrated in Fig. 12, there is no guarantee that $\mathcal{Q}$ is convex. Since $\mathcal{Q}$ can be nonconvex, the optimal server location cannot be determined by formulating the problem as a linear program in general. However in some special cases, the 1-center problem can be formulated as a linear program. We present two such cases as follows:

\section{Case 1: $\mathcal{Q} \cap \mathcal{C}$ is rectangular}

Let us consider a 4-cornered cell $\mathcal{C}$ as shown in Fig. 13 with cell corners $V_{1}, V_{2}, V_{3}$, and $V_{4}$. Let $\mathcal{C}$ be bounded on all sides by gridlines (due to other barriers or users) parallel to the edges of $\mathcal{C}$. In other words, $\mathcal{C}$ is bounded on all sides by 8 rectangular cells labeled Cell $\mathcal{C} 1$, Cell $\mathcal{C} 2$, etc. Let us consider the gridline segments $V_{4} V_{3}$ and $B C$. The new facility

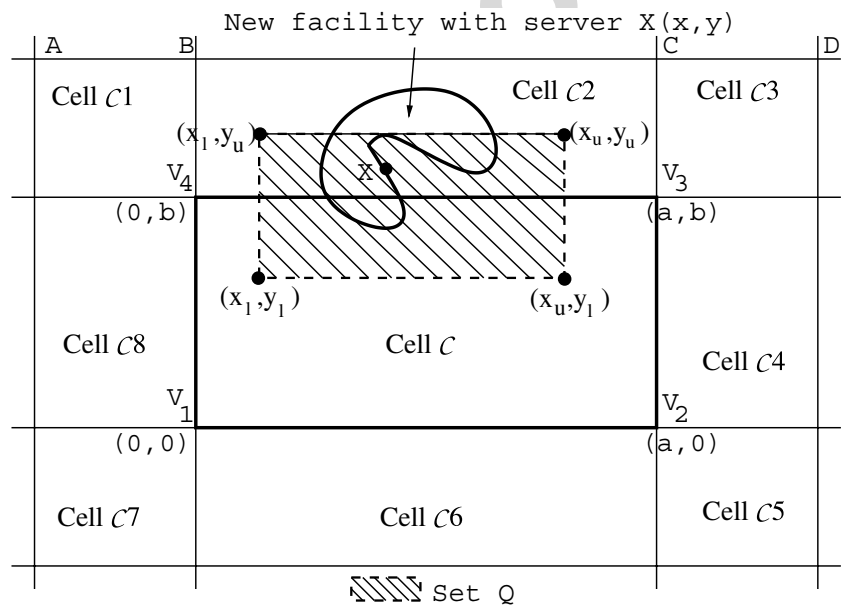

Fig. 13. $\mathcal{Q} \cap \mathcal{C}$ is rectangular. is moved parallel to $V_{4} V_{3}, B C, B V_{1}$, and $C V_{2}$ such that it cuts only $V_{4} V_{3} \in L$. $\mathcal{Q}$ obtained by cutting $V_{4} V_{3} \in L$ is rectangular. Hence $\mathcal{Q} \cap$ Cell $\mathcal{C}$ and $\mathcal{Q} \cap$ Cell $\mathcal{C} 2$ are rectangular.

The rectangular distance from a user $i$ to the server $X$ can be expressed as follows:

$d(i, X)=d\left(i, V_{j}\right)+d\left(V_{j}, H_{k}\right)+d\left(H_{k}, X\right)$,

where $d\left(i, V_{j}\right)$ and $d\left(H_{k}, X\right)$ are constants while $d\left(V_{j}, H_{k}\right)$ is a linear function of the coordinates of the server, i.e., $x$ and $y$. Since $H_{k}, k=1,2,3,4$ and $X$ may not be rectangularly visible, Results 2 and 3 mentioned in Section 3.1 can be utilized to evaluate the distance function $d\left(H_{k}, X\right)$.

Hence the problem to determine the 1-center can be formulated as a linear program over the shaded region $\mathcal{Q} \cap \mathcal{C}$ as follows:

minimize $Z$,

subject to $w_{i}\left[d\left(i, V_{j}\right)+d\left(V_{j}, H_{k}\right)+d\left(H_{k}, X\right)\right]+\gamma_{i} \leqslant Z$

$$
\begin{aligned}
& \text { for } i \in \mathcal{V}_{k}, \forall k, \\
& x_{1} \leqslant x \leqslant x_{\mathrm{u}}, \\
& y_{1} \leqslant y \leqslant y_{\mathrm{u}},
\end{aligned}
$$

where $x_{1}, x_{\mathrm{u}}, y_{1}, y_{\mathrm{u}}$ represent the lower and upper bounds of the $x$-coordinate and $y$-coordinate, respectively, of the $\mathcal{Q} \cap \mathcal{C}$ under consideration and $\mathcal{V}_{k}$ denotes the set of users uniquely assigned to cell corner $V_{k}$, such that $\cup_{k} \mathcal{V}_{k}=D$. A similar linear program can be formulated over $\mathcal{Q} \cap$ Cell $\mathcal{C} 2$.

\section{Case 2: $\mathcal{Q} \cap \mathcal{C}$ is convex polygonal}

If $\mathcal{Q} \cap \mathcal{C}$ is convex polygonal, as illustrated in Fig. $12 \mathrm{~b}, \mathcal{Q}$ can be represented by a set of inequalities 
$A^{\mathcal{Q}}\left(\begin{array}{l}x \\ y\end{array}\right) \leqslant b^{\mathcal{Q}}$.

Hence the problem to determine the 1-center can again be formulated as a linear program, as in Case 1.

\section{Case 3: $\mathcal{Q} \cap \mathcal{C}$ is nonconvex}

However if $\mathcal{Q}$ is nonconvex, the problem to determine the 1-center involves finding the minimax location over a nonconvex set, which can be represented as an optimization problem with a linear objective function and a nonconvex feasible region. Hence the problem can be formulated as a nonlinear program and solved using standard nonlinear programming solvers.

The overall feasible region $F$ is composed of a number of such regions $\mathcal{Q}$. The number and structure of $\mathcal{Q}$ s depends on the number and shapes of barriers. However a number of $\mathcal{Q}$ s will be rectangular or convex polygonal. As demonstrated earlier, the determination of the 1-center for such cases involves solving a linear program. However for nonconvex $\mathcal{Q}$ s, the problem to determine the 1-center can be formulated as a nonlinear program and solved using standard nonlinear programming solvers. Some nonconvex regions might lend themselves to division into a finite collection of convex subsets. Here again, linear programming formulations are possible.

\section{Solution complexity}

Our solution methodology for the fixed orientation problem can be split as two distinct cases: (i) when the facility is fully contained in a cell/subcell (ii) when the facility intersects gridlines. Hence the number of cells/subcells is an important factor that governs our solution complexity. The number of 1 , 2, 3, 4-cornered cells generated depends on the number of barriers, their shapes and their placement. In fact, the number of cells generated is a direct function of the number of points of tangency of each barrier. Formally, $N$ barriers generate at most $C N$ horizontal and $C N$ vertical gridlines where $C$ is a constant that signifies the upper bound on the number of tangency lines of a barrier (in fact, this is our only restriction to the arbitrariness of barriers). Hence the maximal number of 4-cornered cells generated is $\mathrm{O}\left(N^{2}\right)$. However these many 4-cornered cells are typically not generated as gridlines are terminated when they intersect barriers. The number of subcells into which 4-cornered cells can be split is a function of the number of ETTLs generated in each 4-cornered cell. Recall that a user generates at most 1 ETTL in a 4-cornered cell, as proved in [14]. Also since ETTLs between opposing cell corners do not exist, as proved by Lemma 4.2.1, the number of subcells generated is also restricted. Hence $N$ users, one associated with each barrier, can generate $N$ ETTLs in a 4-cornered cell in the worst case, which can intersect in $\mathrm{O}\left(N^{2}\right)$ ways. Hence the number of linear programs to solve is $\mathrm{O}\left(N^{4}\right)$.

When the new facility to be placed intersects gridlines, the number of set $\mathcal{Q}$ s formed depends on the number of gridlines the facility intersects (which in turn is dependent on the number, shapes and relative proximity of the barriers). It is pertinent to note here that the set $\mathcal{Q}$ s are defined when the facility intersects successive horizontal and/or vertical gridlines. The new facility can intersect $C N$ vertical gridlines in $\mathrm{O}\left(N^{2}\right)$ ways because intersecting: (i) one at a time is $C N$ ways, (ii) two at a time is $C N-1$ ways because of the successive intersection requirement, and so on. Similarly, $C N$ horizontal gridlines can be intersected in $\mathrm{O}\left(N^{2}\right)$ ways, and the maximal number of set $\mathcal{Q}$ s is $\mathrm{O}\left(N^{4}\right)$. Note that if a facility cuts one vertical (or horizontal) gridline, $\mathcal{Q}$ can span at most two cells. Similarly if a facility cuts two vertical (or horizontal) gridlines, $\mathcal{Q}$ can span at most three cells. Hence considering only vertical (or horizontal) gridlines, the number of $\mathcal{Q} \cap \mathcal{C s}$ is $\mathrm{O}\left(N^{5}\right)$. Considering there exist both vertical and horizontal gridlines, the number of $\mathcal{Q} \cap \mathcal{C}$ s is $\mathrm{O}\left(N^{6}\right)$. Consequently there are $\mathrm{O}\left(N^{6}\right)$ optimization problems to solve. The complexity of the entire solution procedure would depend on the complexity of the optimization problem in each such set $\mathcal{Q} \cap \mathcal{C}$.

\section{Numerical example}

We now elucidate our solution methodology with a simple five-barrier example, as illustrated by Fig. 4 in Section 3.2. We redraw Fig. 4 as Figs. 14 and 15 for the convenience of the reader. Each barrier has one user. The coordinates of the barrier vertices and users are known. The weight $\left(w_{i}\right)$ and addendum $\left(\gamma_{i}\right)$ of user $i$ are listed in Table 1 .

The new facility (along with its server) is shown in Fig. 14. The first step is to define the feasible region (hatched region as illustrated in Fig. 14). Following the grid construction procedure of Section 3.1 , we draw 11 horizontal (numbered $1-11$ in boxes in Fig. 15) and 13 vertical gridlines (numbered $\mathrm{A}-\mathrm{M}$ in boxes in Fig. 15) through the barrier vertices and 


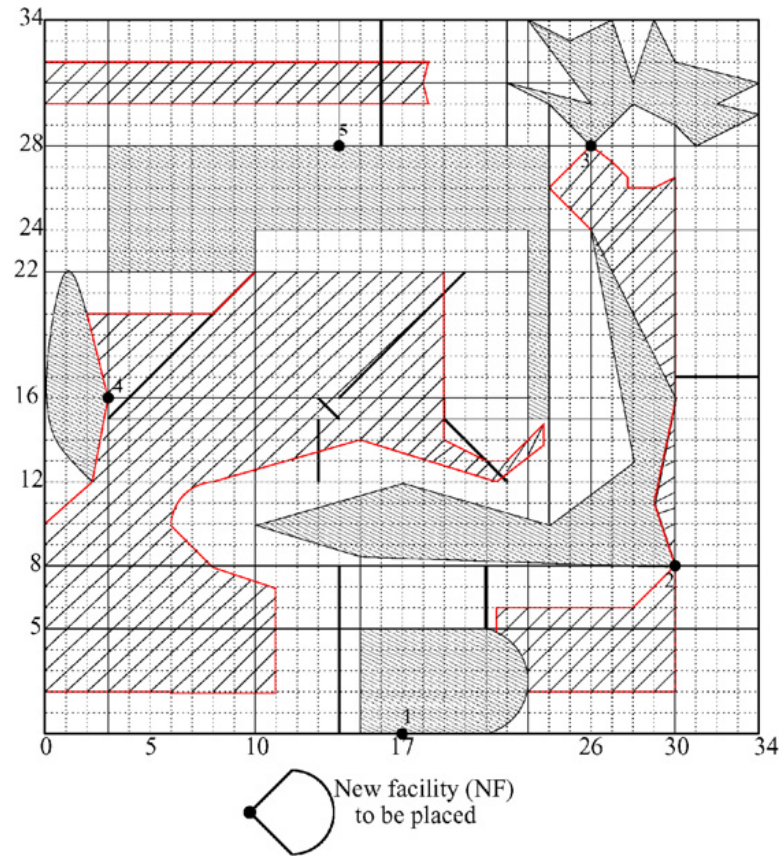

Fig. 14. Feasible region for placing new facility.

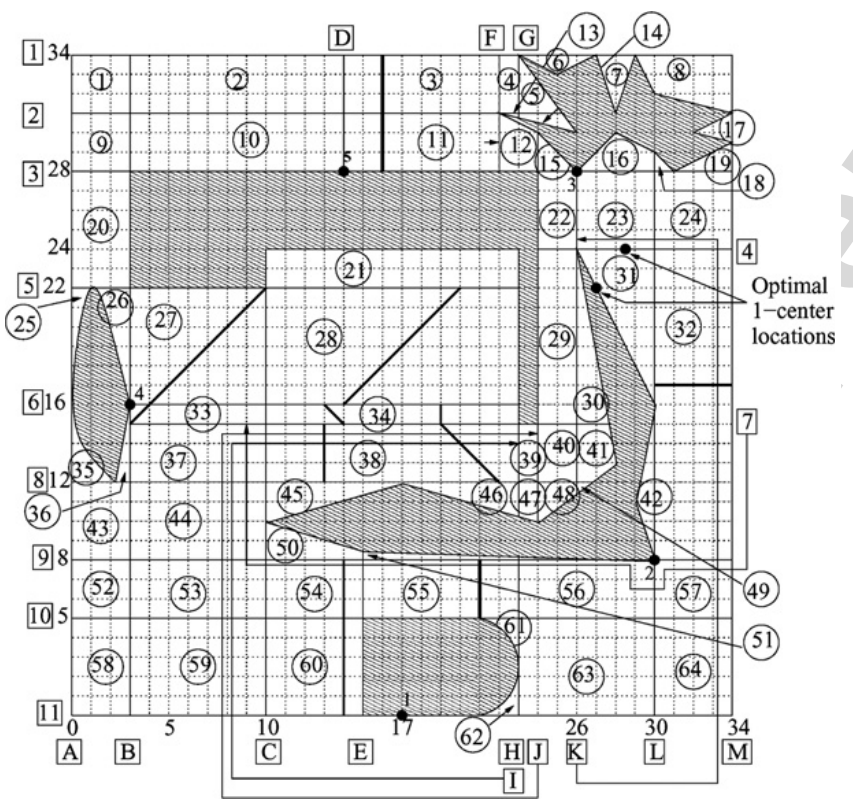

Fig. 15. Cells and gridlines numbered.

Table 1

Example data

\begin{tabular}{llllll}
\hline User $(i)$ & 1 & 2 & 3 & 4 & 5 \\
\hline$w_{i}$ & 1 & 2 & 2 & 1 & 2 \\
$\gamma_{i}$ & 3 & 5 & 2 & 1 & 4 \\
\hline
\end{tabular}

users. As a result, sixty-four cells are generated. The number of 1,2,3 and 4-cornered cells is $0,7,22$ and 35, respectively. In Fig. 15, the cells are numbered in
Table 2

Results when NF is fully contained in cells

\begin{tabular}{llll}
\hline $\begin{array}{l}\text { NF fully contained } \\
\text { in cell }\end{array}$ & \# of sub-problems & $\begin{array}{l}\text { Optimal } \\
\text { 1-center }\left(x^{*}, y^{*}\right)\end{array}$ & $z$ \\
\hline 27 & 1 & $(3,18)$ & 79 \\
28 & 2 & $(10,18)$ & 65 \\
& & $(16,18)$ & 77 \\
32 & 2 & $(30,17)$ & 58 \\
& & $(30,22)$ & 48 \\
59 & 1 & $(6,3)$ & 92 \\
60 & 1 & $(10,3)$ & 94 \\
63 & 1 & $(26,3)$ & 74 \\
64 & 1 & $(30,3)$ & 60 \\
\hline
\end{tabular}

circles. ETTLs generated due to various users are also illustrated.

The new facility (NF) can be fully contained in 7 cells (cells numbered 27, 28, 32, 59, 60, 63 and 64), all of which are 4-cornered. For all these cases, the weighted 1-center problem can be formulated as a linear program (LP). The (local) optimal 1-center location and the corresponding objective function values $(z)$ for all these cases are listed in Table 2.

As an example, let us consider the case when the $\mathrm{NF}$ is fully contained in cell 63 . The weighted 1-center $(x, y)$ objective function for cell 63 is as follows: $\min \max \{[1(6+(x-23)+y)+3],[2(3+(30-x)$

$$
\begin{aligned}
& +(5-y))+5],[2(27+(30-x)+(5-y))+2], \\
& \times[1(31+(x-23)+(5-y))+1], \\
& [2(39+(30-x)+(5-y))+4]\},
\end{aligned}
$$

with feasible server locations being bounded by $23 \leqslant x \leqslant 26$ and $2 \leqslant y \leqslant 3$. This 1 -center problem can be formulated as an LP. The optimal 1-center for cell 63 is $x=26$ and $y=3$ with an objective function value of 74 . Recall that when the NF is fully contained inside cells, ETTLs do not move. Also note that when a cell is split into subcells by an ETTL (for example, cells 28 and 32), the weighted 1-center problem splits into two sub-problems (one for each subcell), each of which can be formulated as an LP.

When the NF intersects gridlines, set $\mathcal{Q}$ s are formed. In this example, 38 such set $\mathcal{Q}$ s are generated. Recall that $\mathcal{Q}$ s may span more than one cell. In all such cases, the weighted 1-center problem has to be solved over $\mathcal{Q} \cap \mathcal{C}$ s. Here, $60 \mathcal{Q} \cap \mathcal{C}$ sets are found. Consequently, there are 60 optimization problems to solve, only one of which turned out to be a nonlinear program (NLP) (case 6 in Table 3). All other cases were formulated as LPs. The LPs 
Table 3

Selected results when NF intersects gridlines

\begin{tabular}{|c|c|c|c|c|c|c|}
\hline Case \# & Lines intersected & Shape of $\mathcal{Q}$ & Shape of $\mathcal{Q} \cap \mathcal{C}$ & LP/NLP & Opt. 1-median $\left(x^{*}, y^{*}\right)$ & $z$ \\
\hline \multirow[t]{2}{*}{1} & $\mathrm{~L}$ & $\begin{array}{l}\text { Mutually disjoint sets, } \\
\text { Convex polygonal (CP) }\end{array}$ & - & LP & $(30,30)$ & 86 \\
\hline & & Rectangular $(\mathrm{R})$ & - & LP & $(27,22)$ & 42 \\
\hline \multirow{2}{*}{2} & $\mathrm{~L}, 4$ & $\mathrm{CP}$ & $\mathrm{R}$ & LP & $(28.67,24)$ & 41.33 \\
\hline & & & $\mathrm{CP}$ & LP & $(26.89,22.22)$ & 41.33 \\
\hline \multirow[t]{2}{*}{3} & $\mathrm{H}, 10$ & Nonconvex & $\mathrm{R}$ & LP & $(23,6)$ & 94 \\
\hline & & Nonpolygonal (NC-NP) & $\mathrm{NC}-\mathrm{NP}$ & LP & $(23,5)$ & 96 \\
\hline 4 & $\mathrm{C}$ & $\mathrm{R}$ & - & LP & $(8.5,3)$ & 87 \\
\hline \multirow[t]{2}{*}{5} & $\mathrm{C}, 9$ & Nonconvex, & $\mathrm{CP}$ & LP & $(8,8)$ & 78 \\
\hline & & Polygonal (NC-P) & $\mathrm{CP}$ & LP & $(8.25,7)$ & 78.5 \\
\hline \multirow[t]{2}{*}{6} & $\mathrm{C}, 8$ & $\mathrm{NC}-\mathrm{NP}$ & $\mathrm{CP}$ & LP & $(6,13)$ & 72 \\
\hline & & & $\mathrm{NC}-\mathrm{NP}$ & NLP & $(8,12)$ & 70 \\
\hline \multirow[t]{3}{*}{7} & $\mathrm{C}, 6,7$ & $\mathrm{R}$ & $\mathrm{R}$ & LP & $(6,15)$ & 68 \\
\hline & & & $\mathrm{R}$ & LP & $(6,15)$ & 68 \\
\hline & & & $\mathrm{R}$ & LP & $(6,16)$ & 70 \\
\hline
\end{tabular}

and NLP were solved using the LP solver LINDO 6.1 and the NLP solver LINGO 8.0. The solution times in all cases were less than a second. We now present a few selected results, when the NF intersects gridlines.

For each of the following cases, Table 3 lists the gridline(s) intersected, shape of $\mathcal{Q}$ s generated, shape of $\mathcal{Q} \cap \mathcal{C}$ (thereby indicating the number of resulting optimization problems), their type (LP or NLP) and the (local) optimal 1-center location along with its objective function value $(z)$.

The optimal 1-center locations (obtained by comparing all local minima) of the new facility are $(26.89,22.22)$ and $(28.67,24.00)$ (Case 2 in Table $3)$, both with objective function values of 41.33 .

We make an interesting observation for Case 3. While one of the $\mathcal{Q} \cap \mathcal{C}$ related to cell 61 is nonconvex nonpolygonal, it is analogous to the 3 -cornered cell for the fully contained case (see Lemma 5.1.3). Thus its boundary dominates the interior and a line search (through an LP) is adequate.

Note that since the new facility intersects gridlines, ETTLs may move. Case 7 in Table 3 is an example in which the ETTLs in cells 26 and 32 move. However the assignment of users to cell corners does not change in any of the cases in this example.

Establishing domination results can significantly reduce the number of optimization problems to be solved. In this example, it can be verified that parts of the feasible region, for example, between (i) $y=$ 30 and $y=32$, (ii) $x=0$ and $x=3$, and (iii) all feasible locations contained strictly inside cell 42 , are dominated. All such feasible locations are excluded from analysis. Moreover, it is worthwhile noting that we can attempt to prune some cells (in which the NF can be fully contained) based on a bound provided by the optimal 1-center location of an infinitesimal facility. The overall efficiency of the solution procedure can thus be further improved.

Finally, the number of optimization problems to be solved depends on the number of barriers, their shapes and relative proximity, as mentioned earlier. However, even for more complex problems, the computational time required to solve each of the mini optimization problems is expected to be negligible.

\section{Conclusions}

In summary, this paper addresses the optimal placement problem of a single finite size arbitrarily shaped facility in the presence of arbitrarily shaped barriers to travel with the center objective. The rectangular travel metric is employed. The server location and the facility orientation define a facility's placement. Due to the complexities associated with the finite size of the new facility, the placement problem with fixed facility orientation is studied in this paper. Based on the subdivision of the feasible region into cells, we obtain cell domination results for 1, 2, 3-cornered cells when the facility is fully contained inside such cells. When the new facility is fully contained in a 4-cornered cell (or a subcell generated by ETTLs), the 1-center placement problem can be formulated as a linear program. When the facility cuts gridlines, we analyze the problem and develop a solution methodology based on distinct but fixed sets of gridlines which the facility intersects. 
Our work can be viewed as an extension of Savas et al. [16] who study a similar problem but with "median" objective and Nandikonda et al. [14] who consider the 1-center placement of an infinitesimal facility. Ref. [16] considers a median objective and proves the objective function to be concave. However since distances are being measured explicitly in our work, the procedure can be applied to a general class of problems irrespective of the objective considered. Unlike [16], we solely consider the user-server interaction. We note that since the new facility to be located itself acts as a barrier to rectangular travel, it can disrupt the level of interaction between two users. Study of the 1-center placement problem under a similar setting but subject to a constraint that the user-user interaction should not exceed a specified threshold is an interesting open problem. Proposing a solution methodology to the variable orientation problem (when the server location $X_{0}$ is known a priori) is also a future research direction.

\section{Acknowledgements}

This work was supported by the National Science Foundation, via grant DMI-0300370. This support is gratefully acknowledged. The authors also wish to acknowledge the help of two anonymous referees, whose comments significantly improved the exposition of this paper.

\section{References}

[1] Y.P. Aneja, M. Parlar, Algorithms for Weber facility location in the presence of forbidden regions and/or barriers to travel, Transportation Science 28 (1) (1994) 70-76.
[2] R. Batta, A. Ghose, U. Palekar, Locating facilities on the Manhattan metric with arbitrarily shaped barriers and convex forbidden regions, Transportation Science 23 (1) (1989) 26-36.

[3] S.E. Butt, T.M. Cavalier, An efficient algorithm for facility location in the presence of forbidden regions, European Journal of Operational Research 90 (1996) 56-70.

[4] P.M. Dearing, H.W. Hamacher, K. Klamroth, Dominating sets for rectilinear center location problems with polyhedral barriers, Naval Research Logistics 49 (7) (2002) 647-665.

[5] Z. Drezner, Facility Location: A Survey of Applications and Methods, Springer-Verlag, NY, 1995.

[6] R.L. Francis, L.F. McGinnis, J.A. White, Facility Layout and Location: An Analytical Approach, Prentice-Hall, Englewood Cliffs, NJ, 1992.

[7] G.Y. Handler, P.B. Mirchandani, Location on Networks, The MIT Press, Cambridge, MA, 1979.

[8] I.N. Katz, L. Cooper, Facility location in the presence of forbidden regions, I: Formulation and the case of euclidean distance with one forbidden circle, European Journal of Operational Research 6 (1981) 166-173.

[9] R.C. Larson, V.O.K. Li, Finding minimum rectilinear distance paths in the presence of barriers, Networks 11 (1981) 285-304.

[10] R.C. Larson, G. Sadiq, Facility locations with the Manhattan metric in the presence of barriers to travel, Operations Research 31 (4) (1983) 652-669.

[11] R.F. Love, J.G. Morris, G.O. Wesolowsky, Facilities Location: Models and Methods, North Holland, NY, 1988.

[12] P.B. Mirchandani, R.L. Francis, Discrete Location Theory, John Wiley and Sons, Inc., New York, 1990.

[13] J.S.B. Mitchell, $l_{1}$ shortest paths among polygonal obstacles in the plane, Algorithmica 8 (1992) 55-88.

[14] P. Nandikonda, R. Batta, R. Nagi, Locating a 1-center on a Manhattan plane with "arbitrarily" shaped barriers, Annals of Operations Research 123 (2003) 157-172.

[15] A. Sarkar, Finite-size facility placement in the presence of generalized congested regions, $\mathrm{PhD}$ thesis, University at Buffalo (SUNY), September 2004.

[16] S. Savas, R. Batta, R. Nagi, Finite-size facility placement in the presence of barriers to rectilinear travel, Operations Research 50 (6) (2002) 1018-1031. 